OPEN ACCESS

Edited by:

Stephan Zipfel,

University of Tübingen,

Germany

Reviewed by:

Jonas Tesarz,

Heidelberg University,

Germany

Florian Junne,

Universitätsklinikum Tübingen,

Germany

*Correspondence:

Johan Siqveland

josi@ahus.no

Specialty section: This article was submitted to

Psychosomatic Medicine,

a section of the journal

Frontiers in Psychiatry

Received: 09 May 2017

Accepted: 22 August 2017

Published: 14 September 2017

Citation:

Siqveland J, Hussain A, Lindstrøm JC, Ruud T and Hauff E (2017) Prevalence of Posttraumatic

Stress Disorder in Persons with Chronic Pain: A Meta-analysis.

Front. Psychiatry 8:164. doi: 10.3389/fpsyt.2017.00164

\section{Prevalence of Posttraumatic Stress Disorder in Persons with Chronic Pain: A Meta-analysis}

\author{
Johan Siqveland ${ }^{1,2,3 *}$, Ajmal Hussain ${ }^{1}$, Jonas Christoffer Lindstrøm ${ }^{4}$, Torleif Ruud ${ }^{1,3}$ and \\ Edvard Hauff ${ }^{3,5}$

\begin{abstract}
${ }^{1}$ Department for Research and Development, Division of Mental Health Services, Akershus University Hospital, Lørenskog, Norway, ${ }^{2}$ Regional Resource Centre for Traumatic Stress and Suicide Prevention, Oslo, Norway, ${ }^{3}$ Institute of Clinical Medicine, University of Oslo, Oslo, Norway, ${ }^{4}$ Health Services Research Unit, Akershus University Hospital, Lørenskog, Norway, ${ }^{5}$ Oslo University Hospital, Oslo, Norway
\end{abstract}

Objective: To summarize evidence for the prevalence of posttraumatic stress disorder (PTSD) among persons with chronic pain (CP).

Methods: We searched databases for studies published between January 1995 and December 2016, reporting the prevalence of PTSD in persons with CP. Two reviewers independently extracted data and assessed the risk of bias. We calculated the pooled prevalence using a random-effects model and performed subgroup analyses according to pain location, the population and assessment method.

Results: Twenty-one studies were included and the PTSD prevalence varied from 0-57\%, with a pooled mean prevalence of $9.7 \%, 95 \%$ Cl (5.2-17.1). In subgroup analysis, the PTSD prevalence was 20.5\%, 95\% Cl (9.5-39.0) among persons with chronic widespread pain, $11.2 \%, 95 \% \mathrm{Cl}(5.7-22.8)$ among persons with headache, and $0.3 \%$, $95 \% \mathrm{Cl}(0.0-2.4)$ among persons with back pain. The prevalence in clinical populations was $11.7 \%, 95 \% \mathrm{Cl}(6.0-21.5)$ and in non-clinical populations 5.1\%, 95\% $\mathrm{Cl}(0.01-17.2)$. In studies of self-reported PTSD symptoms, PTSD prevalence was $20.4 \%, 95 \% \mathrm{Cl}$ (10.6-35.5), and in studies where structured clinical interviews had been used to assess PTSD its prevalence was 4.5\%, 95\% (Cl 2.1-9.3). The risk of bias was medium for most studies and the heterogeneity was high $\left(I^{2}=98.6\right)$.

Conclusion: PTSD is overall more prevalent in clinical cohorts of persons with CP and particularly in those with widespread pain, but may not always be more prevalent in non-clinical samples of persons with $\mathrm{CP}$, compared to the general population. There is a large heterogeneity in prevalence across studies. Future research should identify sources of heterogeneity and the mechanisms underlying the comorbidity of the two conditions.

Keywords: posttraumatic stress disorder, PTSD, chronic pain, systematic review, meta-analysis, prevalence

Abbreviations: CP, chronic pain; PTSD, posttraumatic stress disorder; ICD-10, international classification of diseases 10th revision; DSM IV, diagnostic and statistical manual of mental disorders 4th edition; CI, confidence interval. 


\section{BACKGROUND}

Chronic pain (CP) is among the most common and costly health problems in the West $(1,2)$. A recent epidemiological study in 16 countries (3) found that $19 \%$ of the general population has CP, two-thirds of these describe their pain as moderate and one-third as severe and more women than men report having $\mathrm{CP}$, according to this study. Mental illnesses, such as anxiety disorders and depression, are more prevalent among persons with $\mathrm{CP}(3-5)$, and there is evidence that anxiety disorders could be more strongly associated with painful conditions than depression (6). Mental disorders are associated with more functional impairment in persons with CP (5) and may, therefore, be a negative prognostic factor for recovery (7). So, an improved understanding of the prevalence of mental disorders associated with $\mathrm{CP}$ is important for the planning and dissemination of effective $\mathrm{CP}$ treatment (8). Posttraumatic stress disorder (PTSD) is a relatively common anxiety disorder developing in some people after exposure to traumatizing, unusual events, such as accidents, violence, or war. PTSD is characterized by the three symptom clusters: (i) hyperarousal, (ii) intrusions of memories from the traumatic event, and (iii) avoidance of situations and actions reminiscent of the event (9). The 12-month PTSD prevalence in the general population is reported to be 3.6\% in the United States (10) and 1.1\% in Europe (11). As for CP, there are gender differences in the risk of developing PTSD after exposure to trauma and in the PTSD prevalence. Women have an increased risk of developing PTSD after exposure to both man-made and technological disasters (12), and more women than men have PTSD with an odds ratio (OR) of 2.69 (13). The results of numerous clinical epidemiological studies have led to the conclusion that PTSD is more common in persons experiencing different forms of $\mathrm{CP}$, such as fibromyalgia (FM) (14) and migraine (15). Previous research indicates that PTSD is related to $\mathrm{CP}$ in both clinical samples and samples of the general population. High rates of PTSD have been reported in clinical subjects seeking treatment for $\mathrm{CP}$ (16), and in one study, which included a large sample, it was found that $\mathrm{CP}$ was related to PTSD with an OR of 3.69 (5). Added to this, PTSD comorbidity with CP is a complicating factor for its treatment and results in the efficacy of therapeutic interventions being lower (17).

The reasons for the often-reported association between PTSD and CP are not very well understood, but some theoretical models have been proposed. One model suggest that the relationship is related to a shared vulnerability for developing both PTSD and CP $(18,19)$ while others have suggested that the conditions mutually maintain each other (20) and a recent review summarized possible neurobiological and neuroanatomical models that might explain this association (21).

While the causes for the association between PTSD and other anxiety disorders are still to a large extent unknown, there has been a growing research interest in describing the relationship between mental disorders and somatic syndromes, including CP, and some recent systematic reviews have summarized the available published research. The findings from three previous systematic reviews and meta-analyses represent relevant background material for the present review. A review of the relationship between psychological trauma and functional somatic syndromes (FSS) looked at three $\mathrm{CP}$ disorders: FM, chronic widespread pain (CWP), and temporomandibular disorder (22). The review included a meta-analysis of the results from 71 studies comparing FSS in persons exposed to psychological traumas to non-exposed control individuals and found that exposure to trauma was related to a 2.7 times increase in the risk of developing FSS. The review authors noted that the included studies were of low overall quality and this may have affected the findings and that authors using validated measures of trauma exposure reported a weaker association between trauma exposure and FSS. The association between trauma exposure and FSS, therefore, could be below the pooled estimate from this meta-analysis.

A later study corroborated that there is a positive relationship between exposure to trauma and pain in a meta-analysis of 18 case-control studies into the association between exposure to emotional, physical and sexual abuse, and FM. In this review, a diagnosis of FM was found to be significantly associated with childhood abuse with an OR of 2.49. Finally, in a meta-analysis study, Fishbain et al. (23) analyzed the relationship between PTSD and CP. This review includes 40 studies, and the prevalence of PTSD in them ranged between $0.69 \%$ in a group of persons with lower back pain and $50.1 \%$ in a group of war veterans.

The aims of the present study were to assess the prevalence of PTSD in persons with CP and to determine how it varies according to the location of the pain, the population, and the methods used to assess the diagnosis.

\section{METHODS}

We followed the quality standards for systematic reviews as presented in the PRISMA statement, which is attached as Supplementary material. The review protocol is also attached as Supplementary material.

\section{Search Strategy}

We searched for articles published in the English language after January 1995 and listed in the PsycINFO, MEDLINE, and PubMed databases. January 1995 is when the first studies based on the DSM IV diagnosis handbook could have emerged. The search was last updated in November 2016. We used a number of textword combinations related to $\mathrm{CP}$, such as "chronic pain," "migraine," "chronic daily headache," "fibromyalgia," "widespread pain," "musculoskeletal pain," "rheumatoid pain," "chronic back pain," and "chronic spinal pain" combined with textword for posttraumatic stress "Stress Disorders," "post-traumatic/," and "PTSD." In addition, all the reference lists of the studies included were hand searched for further relevant publications. The search string for the search in the Embase, MEDLINE, and PsycINFO databases is attached as Supplementary material.

\section{Inclusion of Studies}

After eliminating all duplicate studies, two reviewers independently examined the titles and abstracts of all the extracted articles. We retrieved the full texts of articles that were considered, by at least one reviewer, to meet the inclusion criteria. The inclusion criteria were studies published in English reporting the 
point prevalence (within 1 month) of PTSD based on a structured assessment according to the PTSD criteria of the DSM IV or the International Statistical Classification of Diseases and Related Health Problems (10th Edition; ICD-10). Exclusion criteria were military-only samples, samples recruited from a mental healthcare setting, studies using non-structured assessments (such as informal clinical interviews) or assessments that did not represent sufficiently the diagnostic criteria for PTSD. Migraine was considered a CP disorder, based on the assumption that it is a chronic disorder, despite being characterized by episodic presentation of symptoms (24). After retrieving the full texts, the reviewers extensively scrutinized them to confirm the eligibility of the studies based on the above criteria. Any disagreements relating to inclusion eligibility were resolved through discussions.

\section{Data Extraction}

The reviewers (Ajmal Hussain and Johan Siqveland) extracted information about sample size, number of participants with PTSD, pain location, the setting of recruitment, the method of PTSD assessment, and nationality. Both reviewers agreed on the final data extraction, ensuring accuracy, and disagreements concerning data selection were resolved via discussion between them.

\section{Risk of Bias Assessment}

We assessed the risk of bias using an 8-item scale (range 0-8; see Supplementary Material) adapted for this study from the EBMH Notebook list for evaluating risk of bias in prevalence studies (25). This scale comprises various criteria for evaluating sources of bias, such as the quality of the diagnostic assessments and sample size. We gave the studies included one point for each criterion met, with higher scores, therefore, indicating a lower risk of bias. Studies that scored a seven or eight were classified as having a low risk of bias. Those scoring between three and six, and those scoring between 0 and 2, were of medium and high risk, respectively.

\section{Statistical Analysis}

We hypothesized that the prevalence of PTSD would vary according to pain location, assessment method, and population, so we performed three analyses of subgroups. In the first subgroup analysis, studies included were grouped according to pain location: (a) headaches, including migraines, tension-type headaches, chronic daily headaches, and other headaches; (b) back pain, including pain originating from the spine and chronic lower back pain; (c) CWP, according to the American College of Rheumatology criteria of widespread pain or FM syndrome; and (d) other CP conditions including peripheral neuropathic pain or mixed CP. In the second subgroup analysis, we divided studies according to the population studied: (a) participants recruited from clinical settings and (b) participants from non-clinical settings. Finally, in the third subgroup analysis, we grouped the studies according to the assessment method used: (a) self-reporting of assessed PTSD symptoms and (b) a structured clinical interview for assessing a PTSD diagnosis.

As we had expected there to be significant heterogeneity across the studies included, we used a random-effects model. We analyzed the prevalence of PTSD using a binomial-normal random-effects meta-regression model (26) in the "metafor" R package (27). A random-effects model allows for explicit modeling of the heterogeneity of the parameters investigated in each study, assuming that the true logit transformed prevalence rates are normally distributed among the studies. We calculated the 95\% confidence interval (95\% CI) for all estimates. Heterogeneity was assessed and reported using the $I^{2}$ statistic, which describes the variance between studies as a proportion of the total variance. The $I^{2}$ statistic varies between 0 and $100 \%$, with $0 \%$ being interpreted as no heterogeneity, $25 \%$ as low heterogeneity, $50 \%$ as medium heterogeneity, and $75 \%$ and above as a high degree of heterogeneity (28).

We categorized the studies according to pain location, population, and assessment, including them in the model as categorically coded predictors in three separate sub-analyses. Finally, we estimated the regression coefficients and transformed them into prevalence rates for easier interpretation and reporting.

\section{RESULTS}

\section{Overview of Search}

After excluding duplicates, the literature search yielded 1,589 records. In the initial screening of abstracts, 1,473 articles were excluded, as they did not meet the inclusion criteria. After reading the full texts, we excluded 95 studies the reasons for this presented in Figure 1.

The prevalence of PTSD in the studies included is given in Table 1. A forest plot depicting the prevalence of PTSD in the included studies is presented in Figure 2.

Twenty-one articles with a total of 6,750 participants fulfilled the inclusion criteria and were included in the analysis. These 21 studies varied in sample size between 60 patients with migraine (38) and 1,478 patients with orofacial pain (30). Six of the studies included participants with widespread pain, seven studies included participants with headaches, and two studies included participants with back pain. Six studies included participants with other pain conditions or mixed pain. Three structured or semi-structured diagnostic interviews to diagnose PTSD were used in 10 of the studies, with four questionnaires used in 11 studies. All studies made diagnostic categorization according to the DSM IV criteria for PTSD.

\section{Prevalence of PTSD in All Studies}

The pooled prevalence across all studies was 9.7\% (95\% CI $5.2-17.1)$. The lowest prevalence was reported in a populationbased study of persons with lower back pain $(n=110)$, where none of the participants had PTSD (32). In contrast, the highest prevalence was reported in a study of patients with FM $(n=77)$, where $57 \%$ of participants had PTSD (29).

\section{Prevalence of PTSD According to Pain Location, the Study Population, and the Assessment Method Used}

The prevalence rates of PTSD with 95\% CIs and heterogeneity statistics according to the location of the pain, the study population, and the assessment method are presented in Table 2. 


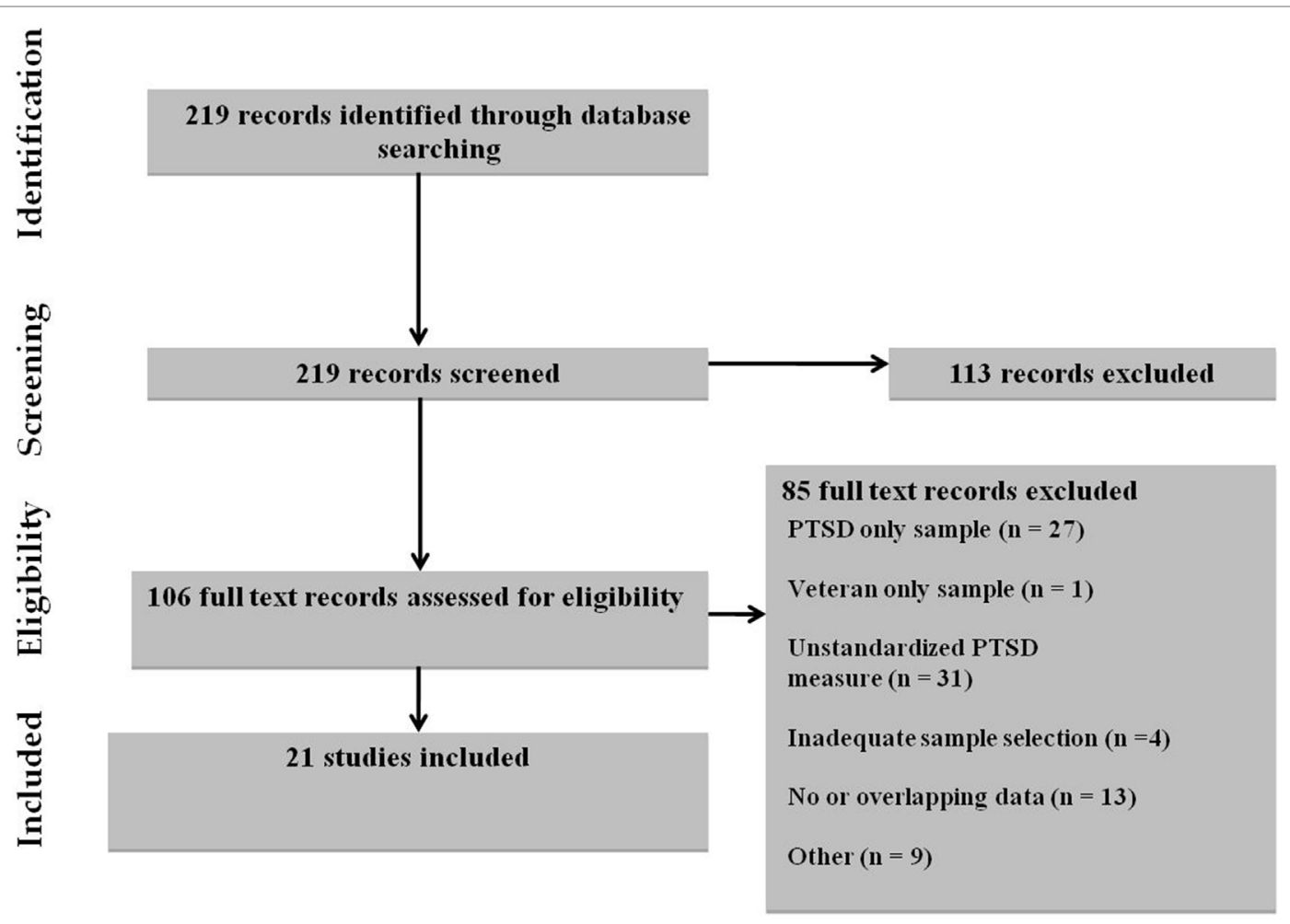

FIGURE 1 | Selection of studies.

The seven studies of persons suffering with headache ( $n=1,555)$ indicated a PTSD prevalence ranging from 2.0 to $28.3 \%$, with a pooled estimate of $11.2 \%$ (95\% CI 5.1-22.8\%). The six studies of persons with CWP $(n=2,189)$ indicated a PTSD prevalence from 4.8 to $57.0 \%$, with a pooled estimate of $20.5 \%$ (95\% CI 9.5-39.0\%). The two studies on persons with back pain $(n=675)$ demonstrated PTSD prevalence rates of 0 and $0.6 \%$, respectively, with a pooled estimate of $0.3 \%$ (95\% CI $0.0-2.4 \%)$. The six studies including other pain conditions or mixed pain conditions reported PTSD prevalences from 1.6 to $29.2 \%$, with a pooled estimate of $9.1 \%$ (95\% CI 3.8-20.5\%).

The 16 studies of clinical samples $(n=4,719)$, reported PTSD prevalences from 0.6 to $57 \%$, pooled estimate $11.7 \%$ (95\% CI 6.0-21.5\%). The five studies with non-clinical samples $(n=2,059)$, reported PTSD prevalences from 0 to $25.7 \%$, pooled estimate $5.1 \%$ (95\% CI 1.4-17.2\%).

The 11 studies where PTSD was assessed with a structured clinical interview $(n=1,790)$ indicated PTSD prevalence rates from 0.6 to $57 \%$, pooled estimate $4.5 \%$ (95\% CI $2.1-9.3 \%$ ). The 10 studies that included self-reported assessments of PTSD ( $n=4,988$ ) reported a PTSD prevalence from 4.8 to $45.8 \%$; pooled estimate $20.4 \%$ (95\% CI $10.6-35.5 \%$ ).

\section{Analysis of Heterogeneity}

The total heterogeneity in the included studies was very high $\left(I^{2}=98.6\right)$. To understand further this large variation in the included we also calculated heterogeneity estimates in the subgroup analysis based on location of pain, population, and assessment method. However, this did not reduce the heterogeneity substantially ( $I^{2}$ 94.2-98.6). Because PTSD is more common in women than it is in men (47), we also analyzed the studies with gender as a co-variate. However, we found that this did not reduce heterogeneity either $\left(I^{2}=98.3\right)$.

\section{Risk of Bias Assessment}

In Table 1, scores for the risk of bias are presented. Of the 21 studies included here, three had a low risk of bias and 18 had a medium risk. The lack of comparison between responders and non-responders, unclear exclusion criteria, and self-reported PTSD symptoms were the most common causes for the higher levels of risk of bias.

\section{DISCUSSION}

The pooled PTSD prevalence across the 21 studies included in the current analysis was $9.7 \%$ (95\% CI 5.2-17.1\%), but varied widely according to pain location, the assessment method, and the sample selection. This finding, however, is in line with a recently published systematic review of PTSD in CP using other selection criteria, which reported a prevalence of $9.8 \%$ (23). Despite the seemingly similar inclusion criteria, 14 out of the 21 studies included in this meta-analysis are unique to the present study and were not included in the study by Fishbain et al. (20). There were several reasons for this low overlap in the included studies in these two reviews. In contrast to the other review article, we only included studies published after the introduction 
TABLE 1 | Selected characteristics, PTSD prevalence, and risk of bias of the included studies.

\begin{tabular}{|c|c|c|c|c|c|c|c|c|}
\hline Reference & Country & Sample selection & Type of pain & $\begin{array}{c}\text { Assessment } \\
\text { instrument }\end{array}$ & $n$ (\% women) & $\begin{array}{c}\text { PTSD } \\
\text { prevalence (\%) }\end{array}$ & Trauma exposure & $\begin{array}{c}\text { Risk of } \\
\text { bias score }\end{array}$ \\
\hline Andersen et al. (16) & Finland and Denmark & Multidisciplinary pain center & Mixed chronic pain (CP) & $\mathrm{HTQ}$ & $304(60.5)$ & 23.0 & $84 \%$ reported $>1$ sign. stressor & 5 \\
\hline Cohen et al. (29) & Israel & Rheumatology clinic & Fibromyalgia (FM) & SCID & 77 (51.9) & 57.0 & Mean exposure 5.7 events & 4 \\
\hline de Leeuw et al. (30) & United States & Orofacial pain center & Orofacial pain & PCL-C & $1,478(86.4)$ & 15.0 & NA & 4 \\
\hline de Leeuw et al. (31) & United States & Orofacial pain center & TTH or migraine & PCL-C & $80(78.8)$ & 16.3 & $64 \%$ reported > 1 sign. stressor & 3 \\
\hline Gerhardt et al. (32) & Germany & General population & Low back pain & SCID & $110(57.3)$ & 0.0 & NA & 6 \\
\hline Ho et al. (33) & China & CP clinic & Mixed CP & SCID & $89(56,2)$ & 4.5 & NA & 6 \\
\hline Häuser et al. (34) & Germany & General population & Widespread pain & PDS & $147(52)$ & 10.9 & $49 \%$ reported $>1$ sign. stressor & 5 \\
\hline Häuser et al. (14) & Germany & General population & $\mathrm{FM}$ & PDS & 395 (93.9) & 45.3 & $74.5 \%$ reported $>1$ sign. stressor & 7 \\
\hline Häuser et al. (35) & $\begin{array}{l}\text { Germany and } \\
\text { United States }\end{array}$ & $\begin{array}{l}\text { Rheumatology clinic/pain } \\
\text { medicine center }\end{array}$ & $\mathrm{FM}$ & PDS & $142(95.8)$ & 33.8 & $60.3 \%$ reported $>1$ sign. stressor & 6 \\
\hline Ifergane et al. (36) & Israel & Headache clinic & Migraine & CAPS & $92(77.2)$ & 6.5 & $16.3 \%$ reported $>1$ sign. stressor & 6 \\
\hline Juang et al. (37) & Taiwan & Headache clinic & $\mathrm{CDH}$ & MINI & $261(80)$ & 2.0 & NA & 6 \\
\hline Karsikaya et al. (38) & Turkey & Neurology clinic & Migraine & SCID & $60(73,3)$ & 28.3 & $63.3 \%$ reported > 1 sign. stressor & 7 \\
\hline Peterlin et al. (39) & United States & Headache center & Migraine & PCL-C & $593(92)$ & 25.0 & $81.2 \%$ reported $>1$ sign. stressor & 6 \\
\hline Proctor et al. (40) & United States & Neurodiagnostic clinic & $\begin{array}{l}\text { Pain related to industrial } \\
\text { injuries }\end{array}$ & CAAPE & $216(51.9)$ & 29.2 & NA & 5 \\
\hline Radat et al. (41) & France & Neurological and pain centers & Neuropathic pain & $\mathrm{MINI}$ & $182(52.2)$ & 3.3 & NA & 7 \\
\hline Raphael et al. (42) & United States & General population & $\mathrm{FM}$ & PCL-C & $1,312(100)$ & 4.8 & $\begin{array}{l}\text { All participants in vicinity of New } \\
\text { York at } 9 / 11\end{array}$ & 4 \\
\hline Reme et al. (43) & Norway & Primary care patients & Low back pain & MINI plus & 565 & 0.6 & NA & 6 \\
\hline Semiz et al. (44) & Turkey & Students & Migraine & SCID & $169(45.7)$ & 3.5 & NA & 6 \\
\hline Smitherman et al. (15) & United States & Students & Migraine & PCL-C & $300(63.1)$ & 25.7 & $\begin{array}{l}69.3 \% \text { reported one or more } \\
\text { significant stressors }\end{array}$ & 4 \\
\hline Taiminen et al. (45) & Finland & Neurology or oral diseases clinic & Mixed CP & SCID & $63(90)$ & 1.6 & NA & 5 \\
\hline Thieme et al. (46) & Germany & Rheumatology department & $\mathrm{FM}$ & SCID & $115(100)$ & 7.8 & $\begin{array}{l}40.9 \% \text { experienced physical abuse } \\
20.9 \% \text { experienced sexual abuse }\end{array}$ & 5 \\
\hline
\end{tabular}

NA, not available; CDH, chronic daily headache; TTH, tension-type headache; HTQ, Harvard Trauma Questionnaire; SCID, structured clinical interview for DSM; PCL-C, posttraumatic checklist civilian version; PDS, Posttraumatic Diagnostic Scale; CAPS, Clinician Administered PTSD Scale; MINI, M.I.N.I. International Neuropsychiatric Interview; CAAPE, comprehensive assessment, and psychological evaluation. 


Study
Andersen et al, 2012
Cohen et al, 2002
de Leeuw, 2005
de Leeuw, 2005
Gerhardt, 2011
Ho, 2011
Häuser et al, 2012
Häuser et al, 2013
Häuser et al, 2015
Ifergane, 2009
Juang, 2000
Karsikaya, 2013
Peterlin, 2009
Proctor, 2013
Radat, 2013
Raphael, 2004
Reme, 2011
Semiz, 2013
Smitherman, 2013
Taiminen, 2011
Thieme, 2004

Type of pain
Other
Widespread
Other
Headache
Back \& neck
Other
Widespread
Widespread
Widespread
Headache
Headache
Headache
Headache
Other
Other
Widespread
Back \& neck
Headache
Headache
Other
Widespread

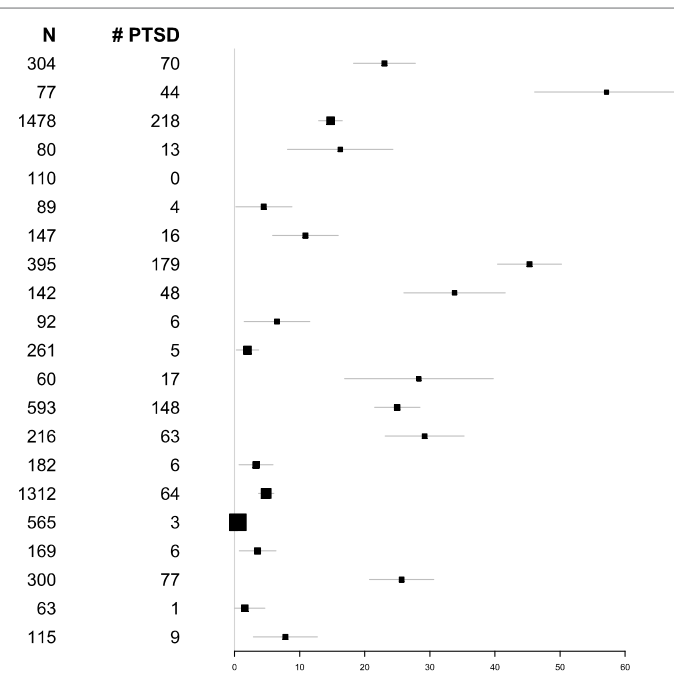

FIGURE 2 | Forest plot of the Prevalence of PTSD Among Persons with Chronic Pain.

TABLE 2 | Pooled estimate and 95\% Cl by location of pain, assessment, and population.

\begin{tabular}{|c|c|c|c|c|c|}
\hline & Cohorts & Participants & $\begin{array}{c}\text { Pooled } \\
\text { PTSD } \\
\text { prevalence }\end{array}$ & $95 \% \mathrm{Cl}$ & $I^{2}(\%)$ \\
\hline \multicolumn{6}{|l|}{ Pain location } \\
\hline Back & 2 & 675 & 0.3 & $0.0-2.4$ & a \\
\hline Head & 7 & 1,555 & 11.2 & $5.1-22.8$ & 96.2 \\
\hline Widespread & 6 & 2,209 & 20.5 & $9.5-39.0$ & 98.0 \\
\hline Other/mixed & 6 & 2,339 & 9.1 & 3.8-20.5 & 97.5 \\
\hline \multicolumn{6}{|l|}{ Assessment } \\
\hline Questionnaire & 10 & 4,988 & 20.4 & $10.6-35.5$ & 94.2 \\
\hline Interview & 11 & 1,790 & 4.5 & $2.1-9.3$ & 97.2 \\
\hline \multicolumn{6}{|l|}{ Sample } \\
\hline Non-clinical & 5 & 2,059 & 5.1 & $1.4-17.2$ & 97.4 \\
\hline Clinical & 16 & 4,719 & 11.7 & $6.0-21.5$ & 98.6 \\
\hline Total & 21 & 6,778 & 9.7 & $5.2-17.1$ & 98.6 \\
\hline
\end{tabular}

aNot applicable due to low number of cohorts.

of DSM IV for more homogenous diagnostic evaluations, we excluded studies recruiting participants from a military setting and we included only studies assessing current PTSD.

Overall, the studies included indicated a higher prevalence of PTSD in persons with CP than that in the general population [between 1 and $4 \%(11,48)$ ]. Here, analysis of the subgroups showed that a higher prevalence of PTSD might be limited to some CP locations and populations, and may possibly be confounded by the assessment methods.

The prevalence of PTSD varied significantly according to the pain location and was most prevalent in persons with widespread pain. The prevalence of PTSD was lowest in the two studies of back pain. The PTSD prevalence in these two studies was not significantly higher than that determined in studies of the general population. The low PTSD prevalence in studies of back pain indicates that back pain may be less closely associated with PTSD, compared to other types of pain. Back pain is the most common type of CP (3) and may be more related to general lifestyle factors rather than to psychological factors. However, there is an alternative, methodological explanation to the low prevalence of PTSD in the back pain studies. The two included studies on back pain both were European diagnostic interview studies with participants from the general population (32) or from primary care (43) rather than from specialist pain clinics. Both assessment type, geographical area for recruiting participants and population are factors related to a lower PTSD prevalence compared to studies based on symptom self-reporting, studies carried out in the USA $(11,48)$ and studies with clinical populations. These three factors might have exaggerated the real difference in the prevalence PTSD between back pain sufferers and those with other types of pain.

Pain that was widespread was the type with the highest prevalence of PTSD. Our analysis was not suited to finding the causes for the differences in the prevalence between different pain types. However, a hypothesis for the differences can be proposed; neurobiological changes related to PTSD in the CNS and endocrine system affect pain sensitivity in the whole body, such as a reduction in the levels of neuropeptide $\mathrm{Y}$, and the neuroactive steroids allopregnanolone and pregnanolone (21). The same underlying pathophysiological processes relating to both $\mathrm{CP}$ and PTSD might be more pronounced in persons with widespread, compared to localized, pain, as pain mechanisms mainly work in a generalized way throughout the body. The relationship between widespread pain and PTSD may also be related to common risk factors. Women are at an increased risk of having both widespread pain and PTSD and so both PTSD and widespread pain are more common in women than in men, with an OR of between 2 and 3 $(13,49)$. This association might be due to the exposure of women to traumatic events with the most serious health consequences. These would be interpersonal, psychological, and physical violence. Lastly, processes maintaining PTSD or pain (or both 
conditions) at the behavioral level may be more pronounced in persons with widespread pain. One possible mechanism is avoidance, which would play a role in mutually maintaining both pain and PTSD (50).

The prevalence of self-reported PTSD was significantly higher than the prevalence of PTSD in studies using clinical interviews for diagnosis, with pooled estimates of 20.4 and $4.5 \%$, respectively. This finding is consistent with that of a previous review on the relationship between somatic disorders and PTSD (51). Since structured or semi-structured diagnostic interviews are the gold standard for PTSD assessments, it is highly likely that this difference is attributable to studies based on self-reporting overestimating the prevalence of PTSD. Questionnaires are often constructed for screening purposes and have cutoffs for probable PTSD diagnosis biased toward sensitivity rather than specificity (52), because this is of the greatest clinical use. The issues with over-inclusiveness may have been further accentuated in these analyses due to the self-reporting questionnaires not being validated for diagnostic accuracy in a CP population. For instance, $\mathrm{CP}$ patients have a higher-than-average level of avoidance and hyperarousal (53), relating to their pain disorder. These symptoms, while they are related to pain, are particularly difficult to differentiate from the symptoms of PTSD, particularly in self-report assessments. Therefore, studies using the self-reporting of PTSD in CP populations may be associated with more false positive PTSD cases, and the prevalence of PTSD reported in interview studies is more comparable to the prevalence from previous interview studies in the general population $(10,11)$. Previous estimates of the PTSD prevalence in CP sufferers may, have considerably overstated this comorbidity.

The data presented here show a clear tendency toward the expected difference between clinical- and population-based samples. PTSD was twice as prevalent in the clinical studies as in the studies of the general population, which was close to being significant. This indicates a putative and interesting difference between clinical and general populations with CP.

\section{Limitations and Risk of Bias}

We applied strict inclusion criteria in terms of only including diagnoses according to the latest diagnostic manuals for mental disorders, which had been available for long enough for studies to have emerged. A later version of the DSM manual for psychiatric diagnosis, DSM 5, was published during the course of this work; however, none of the studies included here involved making diagnostic evaluations according to this newer manual. Despite the attempts to make the inclusion strict to limit heterogeneity between the studies, the meta-analysis showed that the PTSD prevalence varied widely in that there very large amounts of unexplained variance across the studies. Therefore, the pooled mean prevalence has to be interpreted cautiously. The groups included in this review were also heterogeneous in terms of type of pain conditions and degree of clear somatic origin due to the inclusion criteria in the primary studies. The difficulties with finding homogenous associations between PTSD and pain is further hindered by our lack of understanding of the causal relationship between the conditions and more research and model development on this area is clearly needed.

Few of the studies included here gave an adequate description of the recruitment process or rate of non-participation. However, this represents the most common risk of bias in these studies, and so the participants included may not be fully representative of the $\mathrm{CP}$ population. In addition, the number of studies, and hence the participants, included was limited. Even for the most extensively studied pain condition, headaches, only seven studies including 1,555 participants were included. Only articles written in English were included, but as the prevalence of PTSD varies between different countries, adding publications in other languages might have altered our findings. Finally, as some studies included here relied on self-reported $\mathrm{CP}$, not all participants in these studies may have met the formal criteria for a CP disorder.

\section{Clinical Implications}

The identification of mental disorders in persons seeking treatment for CP may be important for several reasons. Pain treatment is often long term and requires good adherence from the patient. A diagnosis of PTSD is related to poor adherence and, therefore, it is important to assess whether this exists alongside the CP (54). The current review indicates that PTSD is prevalent in persons with $\mathrm{CP}$, and particularly among persons seeking treatment for pain, and in those with widespread pain. Clinicians treating $C P$ should be aware of the increased risk of comorbidity with PTSD in their patients, and particularly in patients with widespread pain. In addition to the risk of non-adherence, PTSD is related to an increased risk of substance use disorder (55), so the possibility for dependence needs to be weighed against the benefit for the patient before starting treatment with opioids. Interventions with proven effectiveness in the treatment of both PTSD and CP [such as cognitive behavioral therapy $(56,57)$, somatic experiencing (58), or antidepressants $(59,60)$ ] may be considered as the first treatment in persons with comorbid pain and PTSD.

\section{AVAILABILITY OF SUPPORTING DATA}

All data and material are available upon request to the first author.

\section{ETHICS STATEMENT}

As this was meta-analysis of published data no ethics committee approval was needed.

\section{AUTHOR CONTRIBUTIONS}

JS and EH planned and designed the study, AH and JS screened studies for inclusion and extracted data. JL planned and performed the statistical analysis, and TR participated in drafting the article and revised it critically. All authors have given final approval of the version of the manuscript submitted. 


\section{ACKNOWLEDGMENTS}

The authors wish to thank the personnel at the library at the Akershus University Hospital for kind assistance in locating literature.

\section{REFERENCES}

1. Breivik H, Eisenberg E, O'Brien T. The individual and societal burden of chronic pain in Europe: the case for strategic prioritisation and action to improve knowledge and availability of appropriate care. BMC Public Health (2013) 13:1229. doi:10.1186/1471-2458-13-1229

2. Gaskin DJ, Richard P. The economic costs of pain in the United States. J Pain (2012) 13:715-24. doi:10.1016/j.jpain.2012.03.009

3. Breivik H, Collett B, Ventafridda V, Cohen R, Gallacher D. Survey of chronic pain in Europe: prevalence, impact on daily life, and treatment. Eur J Pain (2006) 10:287-333. doi:10.1016/j.ejpain.2005.06.009

4. Knaster P, Karlsson H, Estlander AM, Kalso E. Psychiatric disorders as assessed with SCID in chronic pain patients: the anxiety disorders precede the onset of pain. Gen Hosp Psychiatry (2012) 34:46-52. doi:10.1016/j.genhosppsych. 2011.09.004

5. McWilliams LA, Cox BJ, Enns MW. Mood and anxiety disorders associated with chronic pain: an examination in a nationally representative sample. Pain (2003) 106:127-33. doi:10.1016/S0304-3959(03)00301-4

6. McWilliams LA, Goodwin RD, Cox BJ. Depression and anxiety associated with three pain conditions: results from a nationally representative sample. Pain (2004) 111:77-83. doi:10.1016/j.pain.2004.06.002

7. Tunks ER, Weir R, Crook J. Epidemiologic perspective on chronic pain treatment. Can J Psychiatry (2008) 53:235-42. doi:10.1177/070674370805300404

8. Turk DC, Wilson HD, Cahana A. Treatment of chronic non-cancer pain. Lancet (2011) 377:2226-35. doi:10.1016/S0140-6736(11)60402-9

9. Association American Psychiatric. Diagnostic and Statistical Manual of Mental Disorders. 4th ed. Washington, DC (1994).

10. Kessler RC, Berglund P, Demler O, Jin R, Merikangas KR, Walters EE. Lifetime prevalence and age-of-onset distributions of distributions of DSM-IV disorders in the national comorbidity survey replication. Arch Gen Psychiatry (2005) 62:593-602. doi:10.1001/archpsyc.62.6.593

11. Darves-Bornoz J-M, Alonso J, de Girolamo G, de Graaf R, Haro J-M, KovessMasfety V. Main traumatic events in Europe: PTSD in the European Study of the Epidemiology of Mental Disorders Survery. J Trauma Stress (2008) 21:455-62. doi:10.1002/jts

12. Neria Y, Nandi A, Galea S. Post-traumatic stress disorder following disasters: a systematic review. Psychol Med (2008) 38:467-80. doi:10.1017/ S0033291707001353

13. Mclean CP, Asnaani A, Litz BT, Hofmann SG. Gender differences in anxiety disorders: prevalence, course of illness, comorbidity and burden of illness. J Psychiatr Res (2011) 45:1027-35. doi:10.1016/j.jpsychires.2011.03.006

14. Häuser W, Galek A, Erbslöh-Möller B, Köllner V, Kühn-Becker H, Langhorst J, et al. Posttraumatic stress disorder in fibromyalgia syndrome: prevalence, temporal relationship between posttraumatic stress and fibromyalgia symptoms, and impact on clinical outcome. Pain (2013) 154(8):1216-23. doi:10.1016/j. pain.2013.03.034

15. Smitherman TA, Kolivas ED. Trauma exposure versus posttraumatic stress disorder: relative associations with migraine. Headache (2013) 53:775-86. doi:10.1111/head.12063

16. Andersen TE, Andersen PG, Vakkala MA, Elklit A. The traumatised chronic pain patient-prevalence of posttraumatic stress disorder - PTSD and pain sensitisation in two Scandinavian samples referred for pain rehabilitation. Scand J Pain (2012) 3(1):39-43. doi:10.1016/j.sjpain.2011.10.001

17. Otis JD, Keane TM, Kerns RD. An examination of the relationship between chronic pain and post-traumatic stress disorder. J Rehabil Res Dev (2003) 40:397-405. doi:10.1682/JRRD.2003.09.0397

18. Asmundson GJG, Katz J. Understanding the co-occurrence of anxiety disorders and chronic pain: state-of-the-art. Depress Anxiety (2009) 26:888-901. doi:10.1002/da.20600

\section{SUPPLEMENTARY MATERIAL}

The Supplementary Material for this article can be found online at http://journal.frontiersin.org/article/10.3389/fpsyt.2017.00164/ full\#supplementary-material.

19. Asmundson GJG, Coons MJ, Taylor S, Katz J. PTSD and the experience of pain: research and clinical implications of shared vulnerability and mutual maintenance models. Can JPsychiatry (2002) 47:930-7. doi:10.1177/070674370204701004

20. Sharp TJT, Harvey AAG. Chronic pain and posttraumatic stress disorder: mutual maintenance? Clin Psychol Rev (2001) 21:857-77. doi:10.1016/S02727358(00)00071-4

21. Scioli-Salter ER, Forman DE, Otis JD, Gregor K, Valovski I, Rasmusson AM. The shared neuroanatomy and neurobiology of comorbid chronic pain \& PTSD: therapeutic implications. Clin J Pain (2015) 31:363-74. doi:10.1097/ AJP.0000000000000115

22. Afari N, Ph D, Ahumada SM, Wright LJ, Ph D, Golnari G, et al. Psychological trauma and functional somatic syndromes: systematic review and metaanalysis. Psychosom Med (2015) 76:2-11. doi:10.1097/PSY.0000000000000010

23. Fishbain DA, Pulikal A, Lewis JE, Gao J. Chronic pain types differ in their reported prevalence of post-traumatic stress disorder (PTSD) and there is consistent evidence that chronic pain is associated with PTSD: an evidence-based structured systematic review. Pain Med (2017) 18:711-35. doi:10.1093/pm/ pnw065

24. Bigal ME, Lipton RB. Concepts and mechanisms of migraine chronification. Headache (2008) 48:7-15. doi:10.1111/j.1526-4610.2007.00969.x

25. Boyle MH. Guidelines for evaluating prevalence studies. Evid Based Ment Health (1998) 1:37-9. doi:10.1136/ebmh.1.2.37

26. Stijnen T, Hamza TH, Özdemir P. Random effects meta-analysis of event outcome in the framework of the generalized linear mixed model with applications in sparse data. Stat Med (2010) 29:3046-67. doi:10.1002/sim.4040

27. Chung SS, Sun K, Bolech CJ. Matrix product ansatz for Fermi fields in one dimension. Phys Rev B Condens Matter Mater Phys (2015) 91:1-48. doi:10.1103/PhysRevB.91.121108

28. Higgins JPT, Thompson SG, Deeks JJ, Altman DG. Measuring inconsistency in meta-analyses. BMJ Br Med J (2003) 327:557-60. doi:10.1136/ bmj.327.7414.557

29. Cohen H, Neumann L, Haiman Y, Matar MA, Press J, Buskila D. Prevalence of post-traumatic stress disorder in fibromyalgia patients: overlapping syndromes or post-traumatic fibromyalgia syndrome? Semin Arthritis Rheum (2002) 32(1):38-50. doi:10.1053/sarh.2002.33719

30. De Leeuw R, Bertoli E, Schmidt JE, Carlson CR. Prevalence of posttraumatic stress disorder symptoms in orofacial pain patients. Oral Surg Oral Med Oral Pathol Oral Radiol Endod (2005) 99(5):558-68. doi:10.1016/ j.tripleo.2004.05.016

31. De Leeuw R, Schmidt JE, Carlson CR. Traumatic stressors and post-traumatic stress disorder symptoms in headache patients. Headache (2005) 45(10): 1365-74. doi:10.1111/j.1526-4610.2005.00269.x

32. Gerhardt A, Hartmann M, Schuller-Roma B, Blumenstiel K, Bieber C, Eich W, et al. The prevalence and type of Axis-I and Axis-II mental disorders in subjects with non-specific chronic back pain: results from a population-based study. Pain Med (2011) 12:1231-40. doi:10.1111/j.1526-4637.2011.01190.x

33. Ho PT, Li CF, Ng YK, Tsui SL, Ng KFJ. Prevalence of and factors associated with psychiatric morbidity in chronic pain patients. J Psychosom Res (2011) 70(6):541-7. doi:10.1016/j.jpsychores.2010.10.006

34. Häuser W, Glaesmer H, Schmutzer G, Brähler E. Widespread pain in older Germans is associated with posttraumatic stress disorder and lifetime employment status - results of a cross-sectional survey with a representative population sample. Pain (2012) 153(12):2466-72. doi:10.1016/j.pain. 2012.09.006

35. Häuser W, Hoffmann EM, Wolfe F, Worthing AB, Stahl N, Rothenberg R, et al. Self-reported childhood maltreatment, lifelong traumatic events and mental disorders in fibromyalgia syndrome: a comparison of US and German outpatients. Clin Exp Rheumatol (2015) 33(20):S86-92. 
36. Ifergane G, Buskila D, Simiseshvely N, Jotkowitz A, Kaplan Z, Cohen H. Posttraumatic stress disorder is not over-represented in a sample population of migraine patients. Eur J Intern Med (2009) 20(2):182-5. doi:10.1016/j. ejim.2008.05.001

37. Juang KD, Wang SJ, Fuh JL, Lu SR, Su TP. Comorbidity of depressive and anxiety disorders in chronic daily headache and its subtypes. Headache (2000) 40(10):818-23. doi:10.1111/j.1526-4610.2000.00148.x

38. Karsikaya S, Kavakci O, Kugu N, Guler AS. Post-traumatic stress disorder and anger in migraine patients. Dusunen Adam J Psychiatry Neurol Sci (2013) 26(4):360-6. doi:10.5350/DAJPN2013260405

39. Peterlin BL, Tietjen GE, Brandes JL, Rubin SM, Drexler E, Lidicker JR, et al. Posttraumatic stress disorder in migraine. Headache (2009) 49(4):541-51. doi:10.1111/j.1526-4610.2009.01368.x

40. Proctor SL, Estroff TW, Empting LD, Shearer-Williams S, Hoffmann NG. Prevalence of substance use and psychiatric disorders in a highly select chronic pain population. J Addict Med (2013) 7(1):17-24. doi:10.1097/ADM. 0b013e3182738655

41. Radat F, Margot-Duclot A, Attal N. Psychiatric co-morbidities in patients with chronic peripheral neuropathic pain: a multicentre cohort study. Eur J Pain (2013) 17(10):1547-57. doi:10.1002/j.1532-2149.2013.00334.x

42. Raphael KG, Janal MN, Nayak S. Comorbidity of fibromyalgia and posttraumatic stress disorder symptoms in a community sample of women. Pain Med (2004) 5(1):33-41. doi:10.1111/j.1526-4637.2004.04003.x

43. Reme SE, Tangen T, Moe T, Eriksen HR. Prevalence of psychiatric disorders in sick listed chronic low back pain patients. Eur J Pain (2011) 15(10):1075-80. doi:10.1016/j.ejpain.2011.04.012

44. Semiz M, Şentürk İA, Balaban H, Yağız AK, Kavakçı Ö. Prevalence of migraine and co-morbid psychiatric disorders among students of Cumhuriyet University. J Headache Pain (2013) 14(1):34. doi:10.1186/1129-2377-14-34

45. Taiminen T, Kuusalo L, Lehtinen L, Forssell H, Hagelberg N, Tenovuo O, et al. Psychiatric (Axis I) and personality (Axis II) disorders in patients with burning mouth syndrome or atypical facial pain. Scand J Pain (2011) 2(4):155-60. doi:10.1016/j.sjpain.2011.06.004

46. Thieme K, Turk DC, Flor H. Comorbid depression and anxiety in fibromyalgia syndrome: relationship to somatic and psychosocial variables. Psychosom Med (2004) 66(6):837-44. doi:10.1097/01.psy.0000146329. 63158.40

47. Lassemo E, Sandanger I, Nygård JF, Sørgaard KW. The epidemiology of post-traumatic stress disorder in Norway: trauma characteristics and preexisting psychiatric disorders. Soc Psychiatry Psychiatr Epidemiol (2017) 52:11-9. doi:10.1007/s00127-016-1295-3

48. Kessler RC, Chiu WT, Demler O, Merikangas KR, Walters EE. Prevalence, severity, and comorbidity of 12-month DSM-IV disorders in the National Comorbidity Survey Replication. Arch Gen Psychiatry (2005) 62:617-27. doi:10.1001/archpsyc.62.6.617

49. Mansfield KE, Sim J, Jordan JL, Jordan KP. A systematic review and metaanalysis of the prevalence of chronic widespread pain in the general population. Pain (2016) 157:55-64. doi:10.1097/j.pain.0000000000000314
50. Villano CL, Rosenblum A, Magura S, Fong C, Cleland C, Betzler TF. The prevalence of post-traumatic stress disorder in chronic pain patients. J Rehabil Res Dev (2007) 44:111-5. doi:10.1682/JRRD.2006.05.0052

51. Afari N, Ahumada SM, Wright LJ, Mostoufi S, Golnari G, Reis V, et al. Psychological trauma and functional somatic syndromes: a systematic review and meta-analysis. Psychosom Med (2014) 76:2-11. doi:10.1097/ PSY.0000000000000010

52. Terhakopian A, Sinaii N, Engel CC, Schnurr PP, Hoge CW. Estimating population prevalence of posttraumatic stress disorder: an example using the PTSD checklist. J Trauma Stress (2008) 21:290-300. doi:10.1002/jts.20341

53. Raphael KG, Natelson BH, Janal MN, Nayak S. A community-based survey of fibromyalgia-like pain complaints following the World Trade Center terrorist attacks. Pain (2002) 100:131-9. doi:10.1016/S0304-3959(02)00273-7

54. Kronish IM, Edmondson D, Goldfinger JZ, Fei K, Horowitz CR. Posttraumatic stress disorder and adherence to medications in survivors of strokes and transient ischemic attacks. Stroke (2012) 43:2192-7. doi:10.1161/ STROKEAHA.112.655209

55. Gielen N, Havermans RC, Tekelenburg M, Jansen A. Prevalence of posttraumatic stress disorder among patients with substance use disorder: it is higher than clinicians think it is. Eur J Psychotraumatol (2012) 3:17734. doi:10.3402/ejpt.v3i0.17734

56. Sturgeon JA. Psychological therapies for the management of chronic pain. Dovepress (2014) 2014:115-24. doi:10.2147/PRBM.S44762

57. Bernardy K, Klose P, Busch AJ, Choy EHS, Häuser W. Cognitive behavioural therapies for fibromyalgia. Cochrane Database Syst Rev (2013) (9):CD009796. doi:10.1002/14651858.CD009796.pub2

58. Andersen TE, Lahav Y, Ellegaard H, Manniche C. A randomized controlled trial of brief somatic experiencing for chronic low back pain and comorbid post-traumatic stress disorder symptoms. Eur J Psychotraumatol (2017) 8:1331108. doi:10.1080/20008198.2017.1331108

59. Puetz TW, Youngstedt SD, Herring MP. Effects of pharmacotherapy on combat-related PTSD, anxiety, and depression: a systematic review and meta-regression analysis. PLoS One (2015) 10:e0126529. doi:10.1371/journal. pone.0126529

60. Hauser W, Bernardy K, Uceyler N, Sommer C, Häuser W, Bernardy K, et al. Treatment of fibromyalgia syndrome with antidepressants: a meta-analysis. JAMA J Am Med Assoc (2009) 301:198-209. doi:10.1001/jama.2008.944

Conflict of Interest Statement: The authors declare that the research was conducted in the absence of any commercial or financial relationships that could be construed as a potential conflict of interest.

Copyright (C) 2017 Siqveland, Hussain, Lindstrøm, Ruud and Hauff. This is an open-access article distributed under the terms of the Creative Commons Attribution License (CC BY). The use, distribution or reproduction in other forums is permitted, provided the original author(s) or licensor are credited and that the original publication in this journal is cited, in accordance with accepted academic practice. No use, distribution or reproduction is permitted which does not comply with these terms. 